\title{
Acute Jejenal Diverticulitis: Case Report of Uncommon Presentation
}

\author{
Hamza Merza Abdulla ${ }^{1, *}$, Ronald Erastus Samuel², Joseph Marc Shabot ${ }^{2}$ \\ ${ }^{1}$ Division of Gastroenterology \& Hepatology, University of Maryland Medical System, Baltimore, Maryland, USA \\ ${ }^{2}$ Division of Gastroenterology \& Hepatology, University of Texas Medical Branch, Galveston, Texas, USA
}

\section{Email address:}

Hamza.Abdulla@umm.edu (H. M. Abdulla), Ronald.e.samuel1@gmail.com (R. E. Samuel), mshabot@utmb.edu (J. M. Shabot)

${ }^{*}$ Corresponding author

\section{To cite this article:}

Hamza Merza Abdulla, Ronald Erastus Samuel, Joseph Marc Shabot. Acute Jejenal Diverticulitis: Case Report of Uncommon Presentation. American Journal of Internal Medicine. Vol. 9, No. 1, 2021, pp. 17-20. doi: 10.11648/j.ajim.20210901.13

Received: November 27, 2020; Accepted: January 12, 2021; Published: January 22, 2021

\begin{abstract}
Jejunal diverticulitis is a rare condition and often difficult to diagnose. We report a case of 68-year-old man with a history of sigmoid diverticulitis who presented to the emergency room with a one day history of acute abdominal pain of the left lower quadrant. Initial CT of the Abdomen and Pelvis revealed ileus vs early/partial small bowel obstruction mildly distended loops of the small bowel with decompressed loops of small bowel in the right lower quadrant with no distinct transition point. The patient was treated with antibiotics for presumed gastroenteritis. However, given persistent symptoms and unclear etiology a CT enterography was ordered and demonstrated jejunal diverticulitis with microperforation and a significant phlegmon surrounding the inflamed diverticulum. The patient was treated with ciprofloxacin and metronidazole and reported complete resolution of symptoms after the course of antibiotics. Jejunal diverticula are extremely rare with an incidence of $0.06 \%$ to $1.3 \%$. Prior case reports described some of its complications including bleeding, perforation and ulceration. Jejunal diverticulitis should be in the differential diagnosis of acute abdomen especially in patients with unclear etiology. This condition can be missed by contrasted CT of the abdomen and dedicated small bowel imaging such as CT Enterography can assist making the diagnosis.
\end{abstract}

Keywords: Jejunum, Diverticulitis, Perforation

\section{Introduction}

Jejunal diverticulitis is a rare etiology of abdominal pain. Jejunal diverticula are herniations of the mucosa and submucosa through the muscular layer of the bowel wall [1, 2]. They are commonly asymptomatic, but can present with widely variable vague symptoms or acute findings such as hemorrhage, obstruction, or perforation [1]. We discuss a case of jejunal diverticulitis complicated with perforation that was missed with initial computer tomography (CT) of the abdomen, but later diagnosed with $\mathrm{CT}$ enterography showing complete resolution of symptoms with antibiotics.

\section{Case Presentation}

A 68-year-old male with a past medical history of coronary artery disease status post coronary artery bypass graft, diabetes mellitus II, hypertension, and sigmoid diverticulitis presented to the hospital one day history of a constant, sudden-onset, sharp, 10/10 in intensity, non-radiating left lower quadrant abdominal pain that is exacerbated by palpation and movement. The pain improved by lying still and associated with nausea, vomiting, and decreased appetite. He denied fever, chills, nausea/vomiting, melena, hematochezia, chest pain, or dyspnea. His vital signs were within normal limits. His physical exam was only remarkable for a soft abdomen that was tender to palpation of left lower quadrant without rebound tenderness or guarding. He had no organomegaly or palpable mass.

First, His complete blood count, basic metabolic panel, and hepatic function panel were within normal limits. A CT scan of the abdomen and pelvis with oral and intravenous contrast showed ileus vs early/partial small bowel obstruction, mildly dilated loops of small bowel with decompressed loops of small bowel in the right lower quadrant with no distinct transition point, a small bowel segment in the left abdomen demonstrates mild wall thickening with surrounding 
mesenteric congestion possibly enteritis, and a small amount of free fluid in the abdomen and pelvis. The general surgery service was subsequently consulted and stated findings were likely gastroenteritis and no acute surgical intervention was needed at that time. Pt was made NPO and maintenance intravenous fluids were started with serial abdominal exams to monitor for worsening of symptoms. Patient's pain slowly improved and was discharged three days later on full liquid diet.

Two weeks after admission, the patient was seen in gastroenterology clinic and complained of epigastric and poorly localized discomfort, early satiety, nausea, and diarrhea/loose stool. Patient stated he was adhering to full liquid diet and pantoprazole. A CT enterography was ordered and showed: jejunal diverticulitis with microperforation and significant phlegmon surrounding the inflamed diverticulum, no abscess in the abdomen, and few scattered diverticula in the jejunum. The patient was subsequently started on ciprofloxacin $500 \mathrm{mg}$ twice daily and metronidazole $500 \mathrm{mg}$ thrice daily for seven days. Pt was seen in clinic two weeks later with complete resolution of symptoms after course of antibiotics.

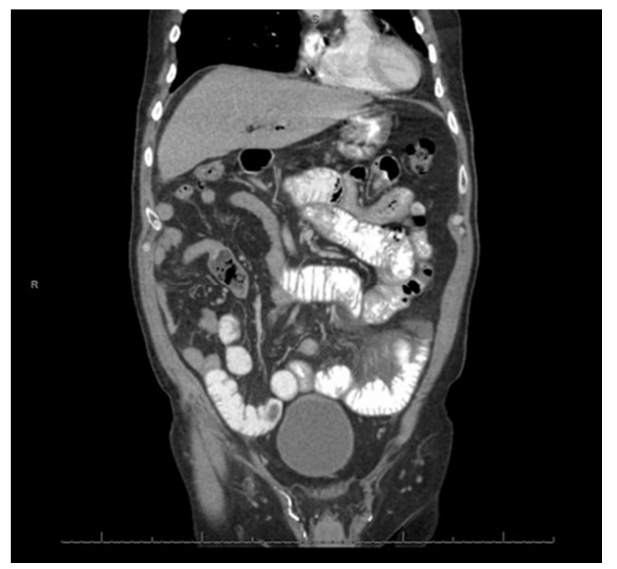

Figure 1. Mildly dilated loops of small bowel with decompressed loops of small bowel in the right lower quadrant.

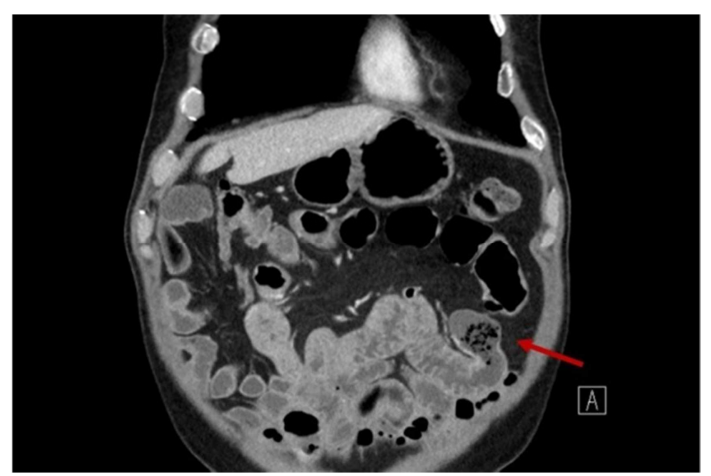

Figure 2. Jejunal diverticulitis with microperforation.

\section{Discussion}

Jejunal diverticula were initially described in 1794 by Soemmering and Baille [3]. The diverticula usually occur in the seventh decade and in the male sex $[4,5]$. Small bowel diverticula in order of decreasing incidence are: duodenal, jejunal, and ileal $[6,10]$.

A study by Krishnamurthy showed three types of abnormalities on microscopy of jejunal diverticula: visceral neuropathy with findings of neuronal and axonal degeneration; visceral myopathy with fibrosis and degenerated smooth muscle cells; progressive systemic sclerosis with fibrosis and decreased numbers of normal muscle cells [14]. The aforementioned abnormalities lead to increased intraluminal pressure $[9,15]$. The diverticula present at the weakest site of the mesenteric border which is where penetration of vasculature occurs in the bowel wall $[8,15]$. The jejunal diverticula are commonly larger and numerous proximally and become smaller and fewer distally $[1,16]$. This is due to weakness of the longitudinal muscle layer and more site of penetration of vasa recta proximally $[17,18]$.

Although $80 \%$ of jejunal diverticula are asymptomatic, they can present with vague symptoms including abdominal pain at any location, nausea, vomiting, loose stools, and bloating [19, 20]. Complications including: chronic abdominal pain, malabsorption, hemorrhage, diverticulitis, obstruction, and perforation, occur in $10-20 \%$ in patients who have jejunal diverticula8, $[12,21]$. Megaloblastic anemia due to vitamin B12 deficiency occurs secondary to bacterial overgrowth and stasis in the diverticulum [22]. Deficiencies occur due to both bile salts and Vitamin B12 being absorbed in the jejunum [23-25]. Malabsorption can present with abdominal pain/discomfort, early satiety, fullness, and diarrhea [26]. It can be treated with antibiotics to eliminate intestinal overgrowth, repletion of nutritional deficiencies, and resection, as a final line of treatment. Small bowel obstruction can present with nausea, vomiting, and abdominal pain [27]. It should be treated with bowel rest and use of a nasogastric tube. The need for further surgical management should be continually assessed in these patients. Hemorrhage can present with melena, hematochezia, and symptoms of anemia. Tagged red blood cell scan or mesenteric arteriography can identify the site of bleed and can be treated with intervascular embolization or surgical intervention1. Jejunal diverticulitis with or without perforation and abscess occur in about $2 \%-6 \%$ of cases [21, 28]. Diverticulitis can also present with abdominal pain, fever, and leukocytosis. It can mimic the presentations of appendicitis, colonic diverticulitis, or cholecystitis [22]. If rebound tenderness is present, perforation of the diverticulitis should be considered [1, 29, 30].

The initial modality of diagnosis is usually abdominal CT with oral and intravenous contrast and the findings can include: air outside the intestinal lumen with a mass lesion, thickened wall with dilated loop of small bowel, edematous surrounding tissues, and in the case of perforation, arrow-head shaped extra-luminal air with surrounding inflammatory findings [9, 22]. In the past, diverticulum were diagnosed with an upper GI series with small bowel follow through or enterolcyis (double contrast of small bowel series) [13]. Double balloon enteroscopy and capsule endoscopy can be considered, but only if patients are stable [21]. If there is still clinical 
suspicion after a CT abdomen/pelvis, CT enterography should be pursued, as it has become the preferred diagnostic study above the modalities previously described [31]. The last diagnostic modality should be laparoscopy or laparotomy due to advances in imaging [32].

The initial treatment for uncomplicated jejunal diverticulitis should be medical management with the antibiotics, ciprofloxacin and metronidazole [7, 30, 33-35]. CT guided aspiration of phlegmon is another option to consider prior to surgical intervention $[11,30]$. If medical treatment does not improve symptoms, segmental jejunal resection with primary anastomosis should be performed with laparoscopy or laparotomy $[9,20,32]$.

\section{Conclusions}

Although, there are prior case reports on the complications related to Jejunal diverticula, this case was unique due to this patient's presentation of initially the small bowel obstruction and subsequently the perforation of the diverticula. There was no evidence of acute abdomen on exams during initial admission or follow-up visit in clinic. This case also shows that initial imaging with CT of abdomen and pelvis did not show any evidence of perforation and it was not until two weeks later that $\mathrm{CT}$ enterography showed the perforation. If clinical suspicion is still present, further imaging should be pursued because basic CT abdomen/pelvis with contrast does not identify all small bowel pathology. The presentation of this case just shows how variable the presentation can be of jejunal diverticulitis and should be considered as a possible differential diagnosis.

\section{References}

[1] de Bree E, Grammatikakis J, Christodoulakis M, et al. The clinical significance of acquired jejunoileal diverticula. Am J Gastroenterol 1998; 93: 2523-8.

[2] Zager JS, Garbus JE, Shaw JP, et al. Jejunal diverticulosis: a rare entity with multiple presentations, a series of cases. Dig Surg 2000; 17: 643-645.

[3] Soemmering ST, Baille M. Anatomie des krankhaften Baues von einigen der wichtigsten Theile in menschlischen Korper. Berline: In Vossiche Buchhandlung 1794.

[4] Osler W. Notes on intestinal diverticula. Ann. anat. Surg 1881; 4: 202-207.

[5] Gordinier H, Sampson J. Diverticulitis (not Meckel's) causing intestinal obstruction. Multiple mesenteric (acquired) diverticula of the small intestine. JAMA 1906; 46: 1585-1590.

[6] Case J. Diverticula of the small intestine other than Meckel's diverticulum. JAMA 1920; 75: 1463-1470.

[7] Staszewicz W, Christodoulou M, Proietti S, et al. Acute ulcerative jejunal diverticulitis: case report of an uncommon entity. World J Gastroenterol 2008; 14: 6265-7.

[8] Kassahun WT, Fangmann J, Harms J, et al. Complicated small-bowel diverticulosis: a case report and review of the literature. World J Gastroenterol 2007; 13: 2240-2.

[9] Kassir R, Boueil-Bourlier A, Baccot S, et al. Jejuno-ileal diverticulitis: Etiopathogenicity, diagnosis and management. Int J Surg Case Rep 2015; 10: 151-3.

[10] Baskin RH, Mayo CW. Jejunal diverticulosis; a clinical study of 87 cases. Surg Clin North Am 1952: 1185-96.

[11] Ackerman NB. Perforated diverticulitis of the terminal ileum. Am J Surg 1974; 128: 426-8.

[12] Akhrass R, Yaffe MB, Fischer C, et al. Small-bowel diverticulosis: perceptions and reality. J Am Coll Surg 1997; 184: $383-8$.

[13] Liu CY, Chang WH, Lin SC, et al. Analysis of clinical manifestations of symptomatic acquired jejunoileal diverticular disease. World J Gastroenterol 2005; 11: 5557-60.

[14] Krishnamurthy S, Kelly MM, Rohrmann CA, et al. Jejunal diverticulosis. A heterogenous disorder caused by a variety of abnormalities of smooth muscle or myenteric plexus. Gastroenterology 1983; 85: 538-47.

[15] Kongara KR, Soffer EE. Intestinal motility in small bowel diverticulosis: a case report and review of the literature. J Clin Gastroenterol 2000; 30: 84-6.

[16] Singal R, Gupta S, Airon A. Giant and multiple jejunal diverticula presenting as peritonitis a significant challenging disorder. J Med Life 2012; 5: 308-10.

[17] Huguenin A, Tirveilliot F, Dell'Erba U, et al. [Acquired jejunal and ileal diverticula (Meckel's excluded)]. Ann Chir 1999; 53: 522-6.

[18] Krummen DM, Camp LA, Jackson CE. Perforation of terminal ileum diverticulitis: a case report and literature review. Am Surg 1996; 62: 939-40.

[19] Altemeier. WA, Bryant LR, Wulsin JH. The surgical significance of jejunal diverticulosis. Arch Surg 1963; 86: 732-45.

[20] Longo WE, Vernava AM. Clinical implications of jejunoileal diverticular disease. Dis Colon Rectum 1992; 35: 381-8.

[21] Sibille A, Willocx R. Jejunal diverticulitis. Am J Gastroenterol 1992; 87: 655-8.

[22] Coulier B, Maldague P, Bourgeois A, et al. Diverticulitis of the small bowel: CT diagnosis. Abdom Imaging 2007; 32: 228-33.

[23] Cooke WT, Cox EV, Fone DJ, et al. The clinical and metabolic significance of jejunal diverticula. Gut 1963; 4: 115-31.

[24] Goldstein F, Wilmer Wirts C, Salen G. Diverticulosis of the Small Intestine Clinical, Bacteriologic, and Metabolic Observations in a Group of Seven Patients Digest Dis Sci 1969; 14: 170 .

[25] Knauer CM, Svoboda AC. Malabsorption and jejunal diverticulosis. Am J Med 1968; 44: 606-10.

[26] Palder SB, Frey CB. Jejunal diverticulosis. Arch Surg 1988; 123: 889-94.

[27] Chiu KW, Changchien CS, Chuah SK. Small-bowel diverticulum: is it a risk for small-bowel volvulus? J Clin Gastroenterol 1994; 19: 176-7. 
[28] Falidas E, Vlachos K, Mathioulakis S, et al. Multiple giant diverticula of the jejunum causing intestinal obstruction: report of a case and review of the literature. World J Emerg Surg 2011; 6: 8 .

[29] Chendrasekhar A, Timberlake GA. Perforated jejunal diverticula: an analysis of reported cases. Am Surg 1995; 61: 984-8.

[30] Novak JS, Tobias J, Barkin JS. Nonsurgical management of acute jejunal diverticulitis: a review. Am J Gastroenterol 1997; 92: 1929-31.

[31] Dave-Verma H, Moore S, Singh A, et al. Computed tomographic enterography and enteroclysis: pearls and pitfalls. Curr Probl Diagn Radiol 2008; 37: 279-87.
[32] Garg N, Khullar R, Sharma A, et al. Total laparoscopic management of large complicated jejunal diverticulum. J Minim Access Surg 2009; 5: 115-7.

[33] Levack MM, Madariaga ML, Kaafarani HM. Non-operative successful management of a perforated small bowel diverticulum. World J Gastroenterol 2014; 20: 18477-9.

[34] Prost A La Denise J, Douard R, Berger A, et al. Small bowel diverticulosis complicated by perforated jejunal diverticula: conservative and/or surgical management? Hepatogastroenterology 2008; 55: 1657-9.

[35] Colvin HS, Kuenfoo C, Rajab TK, et al. Non-surgical management of recurrent perforation of a jejunal diverticulum following previous segmental bowel resection: a case report. J Med Case Rep 2009; 3: 7318. 